\title{
Reprocessamento de instrumental de cirurgia ortopédica em um centro de material e esterilização
}

\author{
Reprocessing with orthopedic surgery instrument in a material and sterilization center \\ Reprocesamiento de instrumentos de cirugía ortopédica en un centro de material y esterilización
}

Recebido: 28/09/2021 | Revisado: 01/10/2021 | Aceito: 07/10/2021 | Publicado: 10/10/2021

\author{
Hedna Maiara Bernardo Pereira \\ ORCID: https://orcid.org/0000-0002-5052-8765 \\ Universidade Federal de Pernambuco, Brasil \\ E-mail: hednamaiara10@gmail.com \\ Constance Majoi Fabricio de Melo \\ ORCID: https://orcid.org/0000-0002-7246-1215 \\ Universidade Federal de Pernambuco, Brasil \\ E-mail: concita.bbp@gmail.com \\ Gésica Kelly da Silva Oliveira \\ ORCID: https://orcid.org/0000-0003-3249-8715 \\ Faculdade do Vale do Ipojuca, Brasil \\ E-mail: gesicakelly.oliveira@gmail.com \\ Gracielly Karine Tavares Souza \\ ORCID: https://orcid.org/0000-0003-4145-2567 \\ Universidade Federal de Pernambuco, Brasil \\ E-mail: gracielly.karine@ hotmail.com \\ Sabrina Emylle Torres Fernandes \\ ORCID: https://orcid.org/0000-0002-4703-4895 \\ Universidade Federal de Pernambuco, Brasil \\ E-mail: sabrinaemylle.torres@gmail.com \\ Nemório Rodrigues Alves \\ ORCID: https://orcid.org/0000-0002-8821-872X \\ Universidade Federal de Alagoas, Brasil \\ E-mail: nemorio_rodrigues@hotmail.com \\ Gabriela Silva Esteves de Hollanda \\ ORCID: https://orcid.org/0000-0002-3216-692X \\ Universidade Federal da Paraíba, Brasil \\ E-mail: gabyhollanda@hotmail.com \\ Nadja da Silva Ferreira \\ ORCID: https://orcid.org/0000-0002-2651-8057 \\ Universidade Federal de Pernambuco, Brasil \\ E-mail: nadjaferreira12@gmail.com \\ Renata Angélica de Oliveira Rosa Mendonça \\ ORCID: https://orcid.org/0000-0002-5556-2348 \\ Universidade Federal de Pernambuco, Brasil \\ E-mail: renata.or@hotmail.com
}

\begin{abstract}
Resumo
Objetivo: Analisar as causas de reprocessamento por não conformidades dos instrumentais de cirurgia ortopédica em um Centro de Material e Esterilização (CME). Método: Trata-se de um estudo descritivo exploratório, retrospectivo, de abordagem quantitativa, desenvolvido em um CME de classe II, a partir do livro de notificação de reprocessamento do serviço e das notificações do software de Gestão de Riscos e Segurança do paciente VIGIHOSP. Resultados: A partir da identificação do quantitativo de repetição de cada não conformidade em caixas específicas, foi possível perceber que a perda da integridade da embalagem é a principal causa de retrabalho. Conclusão: As caixas de cirurgias ortopédica mais reprocessadas estão de acordo com o preconizado nas diretrizes, no entanto, a conformidade, não está evitando a perda da integridade da embalagem.
\end{abstract}

Palavras-chave: Centro esterilização; Esterilização; Gestão.

\footnotetext{
Abstract

Objective: To analyze the causes of reprocessing due to non-conformities of orthopedic surgery instruments in a Material and Sterilization Center (CME). Method: This was a descriptive exploratory, retrospective study with a quantitative approach, developed in a class II MSC, based on the service reprocessing notification book and notifications from the VIGIHOSP Risk Management and Patient Safety software. Results: From the identification of the number of
} 
repetitions of each non-conformity in specific boxes, it was possible to see that the loss of packaging integrity is the main cause of rework. Conclusion: The most reprocessed orthopedic surgery boxes are in accordance with the guidelines recommended, however, compliance is not preventing the loss of packaging integrity.

Keywords: Sterilization center; Sterilization; Management.

\section{Resumen}

Objetivo: Analizar las causas de reprocesamiento por no conformidades de instrumental de cirugía ortopédica en un Centro de Material y Esterilización (CME). Método: Estudio descriptivo, exploratorio, retrospectivo con abordaje cuantitativo, desarrollado en un MSC clase II, basado en el libro de notificaciones de reprocesamiento del servicio y notificaciones del software VIGIHOSP de Gestión de Riesgos y Seguridad del Paciente. Resultados: A partir de la identificación del número de repeticiones de cada no conformidad en cajas específicas, se pudo constatar que la pérdida de integridad del empaque es la principal causa de retrabajo. Conclusión: Las cajas de cirugía ortopédica más reprocesadas están de acuerdo con las pautas recomendadas, sin embargo, el cumplimiento no evita la pérdida de la integridad del empaque.

Palabras clave: Centro de esterilización; Esterilización; Administración.

\section{Introdução}

Centro de Material e Esterilização (CME) é caracterizado como uma unidade de assistência indireta e de apoio técnico para o cuidado ao paciente, sendo destinado ao processamento dos Produtos para Saúde (PPS). De acordo com a complexidade dos produtos para saúde, o CME pode ser classificado em classe I e II, sendo o tipo I aquele que processa produtos não críticos, semicríticos e críticos de conformação não complexa e, o de classe II, o que realiza o processamento de PPS não críticos, semicríticos e críticos de conformação complexa e não complexa (Brasil, 2012).

O Processamento de PPS consiste no conjunto de ações relacionadas a pré-limpeza, recepção, limpeza, secagem, inspeção, preparo, desinfecção ou esterilização, armazenamento e distribuição desses artigos. Com o decorrer dos anos o processamento dos PPS ficou mais criterioso, pois, com a evolução das tecnologias assistenciais, alguns instrumentais tornaramse mais complexos quanto a sua conformação e mais delicados, tornando mais difícil as ações do processamento (Strieder et al., 2019).

Para avaliar a qualidade dos serviços prestados em um CME, são utilizados indicadores de processo, ferramentas que possuem medidas quantitativas e qualitativas para a análise dos serviços oferecidos. $\mathrm{O}$ uso desses indicadores permite avaliar o processo de trabalho da unidade, ajudando no planejamento gerencial, elaboração e execução de atividades para o serviço (Silveira et al., 2015).

Um dos indicadores usados no CME é o retrabalho, que ocorre quando um produto ou serviço é julgado como não conforme em alguma etapa do processamento, ou seja, não atende aos critérios que as normativas vigentes preconizam, tornandoo inadequado para uso pela impossibilidade de assegurar a qualidade do processamento. Com isso, o material será submetido à repetição do processo, de modo a satisfazer os requisitos originalmente especificados, para assim, deixá-lo em condições de ser aceito para uso (Alvim \& Souza, 2018).

As principais não conformidades que levam ao retrabalho no CME são PPS vencidos, resíduo orgânico pósesterilização, etiqueta incorreta, perda ou rasura da etiqueta, pacotes molhados, embalagem não íntegra e resíduo de oxidação. Quando são identificadas não conformidades de forma rotineira, além de denunciar inconsistência na padronização das etapas que compreendem o processamento, ocasionam um aumento no número de reprocessamentos dos PPS, que podem sofrer danos, como desgaste da matéria prima pelos produtos químicos e manuseio. Como resultado os materiais podem quebrar ou formar biofilme, gerando a impossibilidade de seu uso, pelo risco de comprometer o procedimento e oferecer malefícios ao paciente (Lucas et al., 2018).

Os instrumentais cirúrgicos em sua maioria são classificados como críticos. Em algumas especialidades cirúrgicas como a ortopedia, os resíduos insolúveis produzidos são mais difíceis de limpar, pois além de sangue existem restos de ossos. Essas 
dificuldades são agravadas quando os instrumentais são grandes, numerosos e de conformação complexa. Além da limpeza esses materiais oferecem uma dificuldade maior para secagem, esterilização e manutenção da embalagem devido suas características (Souza et al., 2017).

A qualidade do processamento e manutenção da esterilidade dos PPS é a principal missão do CME, pois a ligação direta desses materiais com procedimentos assistenciais em serviços de saúde pode influenciar no surgimento das Infecções Relacionadas à Assistência à Saúde (IRAS), um problema bastante discutido no âmbito da saúde, pois causa o aumento da morbimortalidade dos pacientes, aumentando os gastos na área da saúde. Visto que, a busca pela qualidade do trabalho desse setor vem sendo bastante discutido e monitorado, por possibilitar o planejamento de processos de trabalho mais efetivos para a equipe de enfermagem, possibilitando diminuir a carga de trabalho e ampliar o controle financeiro (Roseira et al., 2017).

Com isso, é imprescindível que o enfermeiro como gestor do CME identifique e analise as causas do retrabalho. Para desenvolvimento do estudo emergiu a seguinte questão norteadora: Quais os fatores modificáveis que concorrem para um reprocessamento de instrumental de cirurgia ortopédica em uma CME? O presente trabalho tem como objetivo analisar as causas de reprocessamento por não conformidades de instrumentais de cirurgia ortopédica em um Centro de Material e Esterilização (CME)

\section{Método}

Trata-se de um estudo descritivo exploratório, retrospectivo, de abordagem quantitativa, desenvolvido no CME de classe II de um hospital escola de grande porte, localizado em Recife, Pernambuco. A coleta ocorreu de março à junho de 2019 após submissão e aprovação do Núcleo de Apoio à Pesquisa (NAP). Esse núcleo é responsável pela análise e deferimento das pesquisas realizadas no hospital.

A coleta apreendeu dados secundários, de modo sequencial, oriundos de notificações de reprocessamento do serviço de instrumentais de cirurgia ortopédica por não conformidade no período de 01 de janeiro de 2019 à 31 de dezembro de 2019, protocolados em livro e em software de Gestão de Riscos e Segurança do paciente (VIGIHOSP). A seleção dos registros obedeceu aos seguintes critérios de inclusão e exclusão: Ser instrumental de cirurgia ortopédica reprocessados por não conformidade; Processados e armazenados no CME do hospital da pesquisa; Está protocolado no livro de notificação de reprocessamento do serviço de armazenamento e distribuição (SAD) do setor da pesquisa; Ter cinco ou mais reprocessamentos no período determinado, visto que, espera-se um total de quatro reprocessamento no ano devido validade por tempo e por isso não é considerado retrabalho; Notificado no Software de Gestão de Riscos e Segurança do paciente (VIGIHOSP). O critério de exclusão foi: Notificação com informações incompletas ou ilegíveis.

Durante a coleta foi realizada análise documental do livro de notificação do SAD e VIGIHOSP. As notificações do livro contêm as seguintes variáveis: identificação do material, quantidade de caixa/bandeja, data e causa do reprocessamento. As causas se subdividem em itens, sendo eles: danificada, vencida e outros. As notificações do livro foram registradas pelos técnicos de enfermagem alocados no arsenal. As notificações do VIGIHOSP são preenchidas pelo setor notificante.

Após preenchimento do instrumento de coleta de dados previamente elaborado, os dados foram digitados em uma planilha do Excel para identificação dos produtos que foram mais reprocessados e a respectiva causa destas ocorrências. A partir dessa identificação foi feita a análise das caixas/bandejas reprocessadas cinco ou mais vezes. Durante a avaliação o material foi pesado, medido, analisado embalagem e etiqueta, armazenamento, quantidade de materiais em cada caixa/bandeja e particularidades como disposição do instrumental dentro da caixa, conformação da caixa e tampa e quantas embalagens foram usadas e o tipo. Essa análise teve o olhar crítico da pesquisadora que buscou na literatura as orientações sobre embalagem e preparo de caixas cirúrgicas. 


\section{Resultados}

No total foram identificados 140 PPS de cirurgia ortopédica notificados no livro de protocolo do SAD para reprocessamento por não conformidade.

A Tabela 1 dispõe o quantitativo de reprocessamentos notificados no protocolo do SAD. Além do percentual total a tabela demonstra o quantitativo de cada não conformidade identificada no material de cirurgia ortopédica.

Tabela 1 - Motivo das ocorrências de reprocessamentos por não conformidades dos materiais de cirurgia ortopédica notificados, hospital escola, Recife - PE, 2019.

\begin{tabular}{ccc}
\hline Motivo dos reprocessamentos & Número & $\%$ \\
\hline Validade vencida & 91 & 29,6 \\
\hline Embalagem danificada & 196 & 63,8 \\
\hline Outros & 3 & 0,9 \\
\hline Dados perdidos & 17 & 5,5 \\
\hline Quantitativo de reprocessamento & 307 & $100 \%$ \\
\hline
\end{tabular}

Fonte: Dados da pesquisa.

Ao todo foram identificadas 16 caixas de cirurgia ortopédicas reprocessadas cinco ou mais vezes por não conformidade, no entanto, só foi possível analisar 08, pois uma delas fazia parte dos consignados que foram recolhidos pela empresa responsável e sete foram incluídas no critério de exclusão, uma vez que, não continha a descrição completa do volume na notificação, inviabilizando a identificação da caixa reprocessada.

Na Tabela 2 pode-se observar os instrumentais de cirurgia ortopédica que foram analisados, o número de reprocessamento e as não conformidades que ocorreram com cada caixa analisada.

Tabela 2 - Materiais de cirurgia ortopédica segundo o quantitativo e motivo dos reprocessamentos, hospital escola, Recife - PE, 2019.

\begin{tabular}{|c|c|c|c|c|}
\hline Material & $\begin{array}{l}\text { Número de } \\
\text { reprocessamento }\end{array}$ & $\begin{array}{c}\text { Perda da } \\
\text { integridade }\end{array}$ & Vencido & Outros \\
\hline $\begin{array}{l}\text { Instrumental fixação de coluna } \mathrm{P} / \text { placa } \\
\text { cervical }\end{array}$ & 7 & 6 & 1 & - \\
\hline Instrumental cervical 3/3 (consignados) & 10 & 8 & 2 & - \\
\hline Quadril não cimentado $1 / 2$ & 7 & 7 & & - \\
\hline Caixa de placa cervical anterior & 5 & 5 & & - \\
\hline $\begin{array}{l}\text { Instrumental coluna lombar } 1 / 2 \\
\text { (consignados) }\end{array}$ & 6 & 5 & 1 & - \\
\hline Conjunto gaiola toracolombar & 5 & 4 & 1 & - \\
\hline $\begin{array}{l}\text { Bandeja grandes fragmentos } 4,5 \\
\text { implantes e placa } 2 / 3\end{array}$ & 5 & 5 & & - \\
\hline $\begin{array}{l}\text { Caixa de instrumental para implante } \\
\text { MINIMICRO (consignados) }\end{array}$ & 6 & 6 & & - \\
\hline TOTAL: & 51 & 46 & 5 & \\
\hline
\end{tabular}

Fonte: Dados da pesquisa. 
A partir da identificação do quantitativo de repetição de cada não conformidade em caixas específicas, foi possível perceber que a perda da integridade da embalagem é a principal causa de retrabalho com instrumentais de cirurgia ortopédica, seguida pela perda da validade.

A data de validade dos materiais do CME da pesquisa é de três meses para grau cirúrgico e SMS ambas são embalagens usadas na esterilização a vapor. Seguindo essa determinação, o material, se não usado em algum momento, obrigatoriamente irá ser reprocessado quatros vezes no ano, por isso, pensando no contexto de retrabalho, os que foram reprocessados apenas quatro vezes não entraram para análise. O quantitativo de reprocessamento entre os materiais analisados não teve muita desproporção, pois o número de reprocessamento ficou entre 5 e 10.

As dimensões e peso das caixas analisadas são descritos no Quadro 1. Houve variações entre os valores encontrados, no entanto, apenas a de quadril não cimentado $1 / 2$ e conjunto gaiola tóracolombar foram não conforme no comprimento, ultrapassando dois e três centímetros respectivamente, do valor preconizado pelas normativas vigentes.

Quadro 1 - Dimensões e peso das caixas com os materiais de cirurgia ortopédica analisados, hospital escola, Recife - PE, 2019.

\begin{tabular}{|l|c|c|c|c|c|}
\hline Material & Comprimento & Largura & Altura & $\begin{array}{l}\text { Peso } \\
(\mathbf{k g})\end{array}$ & $\begin{array}{c}\text { Quantidade de } \\
\text { peças }\end{array}$ \\
\hline $\begin{array}{l}\text { Instrumental fixação de coluna } \\
\text { P/ placa cervical }\end{array}$ & $48 \mathrm{~cm}$ & $25,3 \mathrm{~cm}$ & $8 \mathrm{~cm}$ & 5,205 & 10 \\
\hline $\begin{array}{l}\text { Instrumental cervical 3/3 } \\
\text { (consignados) }\end{array}$ & $42,5 \mathrm{~cm}$ & $28 \mathrm{~cm}$ & $1,5 \mathrm{~cm}$ & 4,215 & 33 \\
\hline Quadril não cimentado 1/2 & $57 \mathrm{~cm}$ & $33 \mathrm{~cm}$ & $12 \mathrm{~cm}$ & 7,400 & 21 \\
\hline Caixa de placa cervical anterior & $25 \mathrm{~cm}$ & $22 \mathrm{~cm}$ & $5,5 \mathrm{~cm}$ & 1,560 & 78 \\
\hline $\begin{array}{l}\text { Instrumental coluna lombar 1/2 } \\
\text { (consignados) }\end{array}$ & $41,5 \mathrm{~cm}$ & $28 \mathrm{~cm}$ & $11,5 \mathrm{~cm}$ & 4,305 & 26 \\
\hline $\begin{array}{l}\text { Conjunto gaiola toracolombar } \\
\text { Bandeja grandes fragmentos 4,5 } \\
\text { implantes e placa 2/3 }\end{array}$ & $58 \mathrm{~cm}$ & $30 \mathrm{~cm}$ & $11,5 \mathrm{~cm}$ & 4,795 & 24 \\
\hline $\begin{array}{l}\text { Caixa de instrumental para } \\
\text { implante } \\
\text { (consignados) }\end{array}$ & $43 \mathrm{~cm}$ & $21,5 \mathrm{~cm}$ & $10,5 \mathrm{~cm}$ & 5,890 & 21 \\
\hline
\end{tabular}

Fonte: Dados da pesquisa.

Para a análise do VIGIHOSP foi realizada a verificação de todas as notificações que o CME recebeu no ano de 2019. Obteve-se um total de vinte, desses, apenas duas notificações eram referentes ao material de ortopedia e por isso esses instrumentais não passaram por análise.

\section{Discussão}

Para garantir a eficácia do processo de esterilização são utilizados indicadores físicos, químicos e biológicos para monitorização de cada ciclo. Após estéril, os cuidados com os materiais continuam e a manutenção da esterilidade dos produtos é a principal missão dessa etapa do processamento (Queiroz et al., 2019).

As embalagens, conhecidas como sistema de barreira estéril, além de permitir a esterilização do conteúdo, mantém o material estéril, pois elas protegem de possíveis contaminações e permite a apresentação estéril do produto. Para a embalagem exercer sua função ela precisa estar íntegra e em um ambiente que tenha condições adequadas, sendo capaz de manter o material 
estéril mesmo após sair do ambiente controlado. Contudo, o tempo de proteção diminui, à medida que o material é exposto a risco de contaminação em um novo ambiente de armazenagem ou transporte, fora das condições ideais (Queiroz et al., 2019).

Para que seja assegurado práticas seguras com os PPS existem normativas que determinam as características da estrutura física para armazenamento, particularidades do clima, circulação de pessoas, fluxo e a forma correta de manipulação e transporte do material. Essas orientações tem o intuito de manter a esterilidade do material (SOBECC, 2017).

Todas essas práticas após esterilização têm como objetivo evitar eventos relacionados (events related), definidos como comportamentos adversos ou condições ambientais que podem levar a perda da integridade das embalagens e resultar na contaminação dos PPS. São considerados eventos relacionados: o número de vezes que um produto é manuseado antes do uso, situações inadequadas durante o transporte, queda do produto no chão, abrir e fechar a embalagem, carregar o produto juntos ao corpo, prender com barbante ou elásticos, guardar em gavetas apertadas e empilhamentos (Freitas et al., 2015).

Estes eventos relacionados são os principais aspectos que o gestor do CME deve usar para definir a data de validade dos seus PPS, já que cada instituição possui suas particularidades. O responsável técnico é o maior conhecedor das limitações e potenciais do seu serviço. Seguindo essa linha de pensamento, as normativas vigentes preconizam que a data de validade dos PPS estéreis é definida por cada instituição de serviço (Freitas et al., 2015).

As diretrizes atuais também preconizam as dimensões e o peso que as caixas e pacotes devem seguir. O peso não deve ultrapassar $11 \mathrm{~kg}$ e suas dimensões máximas devem ser de 55x33x22cm. Essas recomendações buscam preservar a saúde ocupacional dos colaboradores e facilitar a entrada do vapor no material para esterilização (SOBECC, 2017; Freitas et al., 2015).

Outra recomendação forte é a divisão de caixas que contenham muitas bandejas. Elas devem ser esterilizadas separadamente, e assim, ter uma secagem e esterilização mais eficiente. Essa orientação é seguida pelo CME da pesquisa, pois existem diversas caixas de cirurgia ortopédica subdivididas. É possível perceber que essa divisão também ajuda no cumprimento das recomendações de peso, pois os instrumentais e implantes são pesados e sem a divisão o peso seria maior, podendo levar a não conformidade.

$\mathrm{Na}$ análise dos materiais, foi verificado que todas as caixas de cirurgia ortopédica são embaladas com manta de polipropileno, conhecida como tecido não tecido [spunbonded /meltblown/ spunbonded (SMS)]. Essa embalagem é descartável e confere como benefícios a resistência mecânica e microbiana além de estar disponível no mercado com diferentes resistências e tamanhos (SOBECC, 2017).

De acordo com a SOBECC (2017), a gramatura do SMS deve ser escolhida a partir do peso e conformação do material. Orienta-se o uso de dupla embalagem ou a versão mista com fibra sintética em caixas cirúrgicas pesadas e em materiais pontiagudos. Outra recomendação é a proteção dos cantos vivos e saliências na base externa das caixas, para assim diminuir o risco de dano ao SMS durante o manuseio (SOBECC, 2017).

Todas as caixas analisadas possuíam pelo menos um desses itens que podem levar a perda da integridade da embalagem durante o manuseio. Outro ponto observado é que todas as caixas eram embaladas com duas mantas e um tecido de algodão para proteger cantos vivos. Porém, mesmo com essas proteções extras as caixas analisadas perderam a integridade da embalagem antes da validade por tempo.

As Figuras 1 e 2 apresentam inconformidades. A caixa de grandes fragmentos 4,5 implantes e placas 2/3 faz parte de uma caixa que foi subdividida. Durante a análise, foi observado que o sistema de barreira estéril não estava íntegro e faltava cerca de um mês para o prazo limite, determinado como validade. Essa caixa tem uma base composta por cantos vivos e quando exposta aos eventos relacionados ocorre a perda da integridade da embalagem e consequentemente da esterilidade do material. 
Figura 1 - Bandeja grandes fragmentos 4,5 implantes e placa 2/3- embalagem rasgada.

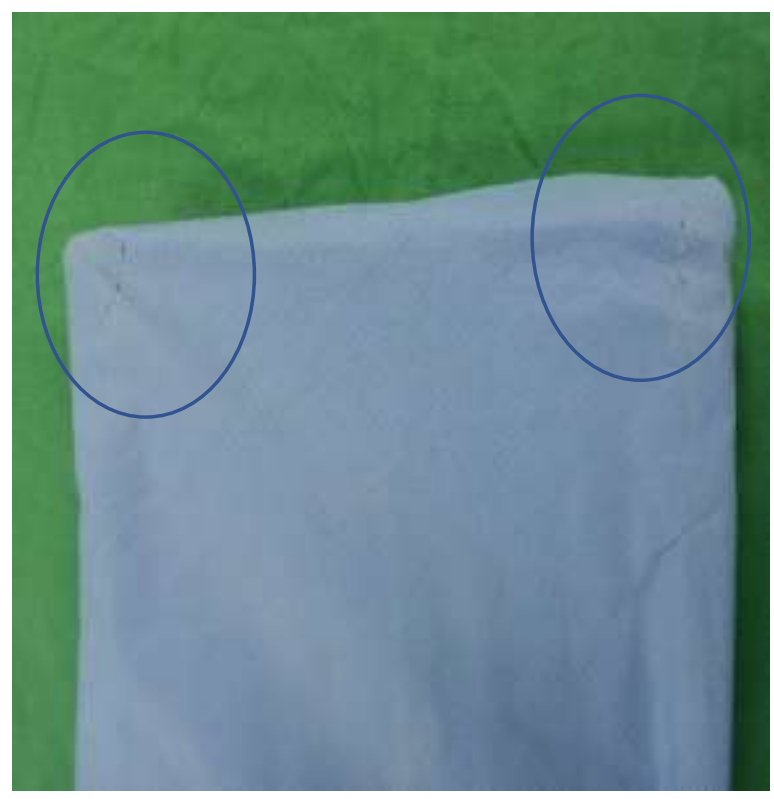

Fonte: Acervo pessoal dos autores.

Figura 2 - Bandeja grandes fragmentos 4,5 implantes e placa 2/3 - sem embalagem.

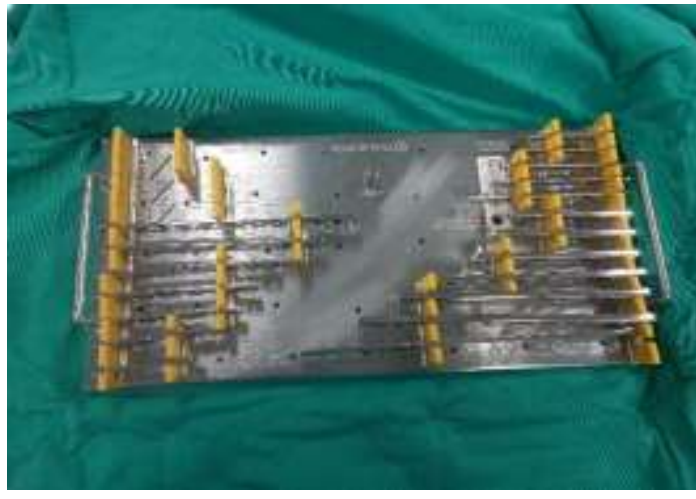

Fonte: Acervo pessoal dos autores.

O sistema de barreira estéril, bandejas, caixas e etiquetas eram considerados PPS e por isso era regulado pela GerênciaGeral de Tecnologia de Produtos para Saúde (GGTPS). Essa regulamentação oferecia uma segurança maior em relação a qualidade desses produtos. No entanto, a nota técnica n 218/2020/SEI/GEMAT/GGTPS/DIRE3/ANVISA retirou esses itens da lista dos PPS e por isso, não são regulados pela GGTPS/ ANVISA desde essa atualização.

Essa mudança na regulação pode refletir na qualidade desses produtos, pois a diminuição da fiscalização poderá acarretar na diminuição da qualidade das embalagens e com isso, o aumento da perda da integridade e consequentemente a elevação do retrabalho e maior custo.

\section{Conclusão}

O reprocessamento das caixas de cirurgia ortopédica, de uma CME de um hospital escola em Recife, está de acordo com o preconizado nas diretrizes, no entanto, a conformidade, não está evitando a perda da integridade da embalagem. Esse resultado nos remete aos eventos relacionados que são a provável causa do retrabalho por perda da integridade das embalagens no CME do estudo, podendo está relacionada a qualidade na resistência da embalagem e ao manuseio inadequado dos materiais esterilizados. 
Durante o processo de coleta algumas limitações complicaram o andamento da pesquisa, dentre estas, o livro de notificação, que era manuscrito, com caligrafias de difícil compreensão. Soma-se a isso, a falta de notificações completas das caixas cirúrgicas reprocessadas, impossibilitando a identificação do volume, uma vez que, o material notificado fazia parte de um conjunto de volumes. Esse problema levou a não análise de 07 caixas que foram reprocessadas 05 ou mais vezes.

A partir dessa limitação recomenda-se a elaboração de uma notificação mais prática, didática e que leve ao preenchimento completo das informações. Uma sugestão é o desenvolvimento de um livro com itens digitados e com espaço especifico e separados para identificar nome e o número do volume da caixa. Além da atualização do livro de notificação do SAD é necessário que gestão do CME oriente a equipe sobre a importância da notificação correta no livro, visto que, é uma forma de respaldo dos profissionais para resolução de situações em que a notificação esclarecerá dúvidas. Outro ponto importante são os indicadores que o livro fornece que só serão válidos para o gestor se estiveram completos.

Os resultados obtidos nesse estudo podem subsidiar a orientação sobre a importância das notificações e do seguimento dos procedimentos operacionais padrão desenvolvidos pela gestão para a obtenção de indicadores de qualidades satisfatórios.

Diante dessas conclusões, compreende-se a necessidade do desenvolvimento de um estudo observacional para uma melhor identificação e análise dos eventos relacionados que os PPS são submetidos, para assim, desenvolver estratégias que evitem ou minimizem a ocorrência deles.

\section{Referências}

ANVISA. Agência Nacional de Vigilância Sanitária. Nota Técnica No 218/2020/SEI/GEMAT/GGTPS/DIRE3/ANVISA. Atualização da lista de produtos não regulados pela GGTPS. http://portal.anvisa.gov.br/registros-e-autorizacoes/produtos-para-a-saude/produtos-nao-regulados.

Alvim A. L. S., \& Souza, K. F. Causas de retrabalho de produtos para Saúde no centro de materiais e esterilização. Revista Sobecc. 23(1): 3-6. https://revista.sobecc.org.br/sobecc/article/view/263.

Brasil. Agencia Nacional de Vigilância Sanitária. Resolução da Diretoria Colegiada- RDC $N^{\circ} 15$, de 15 de março de 2012. http://bvsms.saude.gov.br/bvs/saudelegis/anvisa/2012/rdc0015_15_03_2012.html.

Freitas, L. R., Tiplee, A. F. V., Pires, F. V., Melo, D. S. et al. (Lack of) care for sterilized healthcare products during transport to and storage in inpatient units. Texto \& Contexto - Enfermagem. 24(1):253-262. http://dx.doi.org/10.1590/0104-07072015003550013.

Lucas, T. C., Souza, M. X., Guedes, H. M., Braga, E. V. O. et al. Identificação de deteriorações físicas e químicas nos instrumentais cirúrgicos após reprocessamentos. Rev de Enfermagem do Centro-Oeste Mineiro. http://www.seer.ufsj.edu.br/index.php/recom/article/view/1926

Queiroz, M. Y. K., Martins, C. C., Mendes, E. C. B., \& Kosusni, D. I. Prazo de validade de esterilização de artigos utilizados em uma clínica odontológica. Rev Nursing 22(250): 2794-2799. https://pesquisa.bvsalud.org/portal/resource/pt/biblio-998160

Roseira, C. E., Mariano, D. S., Passos, I. P. B. D., Orlandi, F. S. et al. Praticabilidade de indicadores validados para o processamento de produtos para saúde. Rev enferm UFPE on line. 11(6): 2541-7. https://periodicos.ufpe.br/revistas/revistaenfermagem/article/view/23422/19104.

Silveira, T. V., Prado Junior, P. P., Siman, A. G., \& Amaro, M. O. F. The importance of using quality indicators in nursing care. Rev Gaúcha de Enfermagem. 36(2):82-88. https://www.scielo.br/scielo.php?pid=S1983-4472015000200082\&script=sci_abstract.

SOBECC. Associação Brasileira de Enfermeiros de Centro Cirúrgico Recuperação Anestésica e Centro de Material e esterilização (SOBECC). Diretrizes de práticas em enfermagem cirúrgica e processamento de produtos para saúde. (7a ed.), SOBECC/Barueri: Manole; 2017.

Souza, R. Q., Bronzatti, J. A. G., Laranjeira, P. B., Minica, L. M. J. et al. Avaliação da segurança do processamento de fresas intramedulares flexíveis Para cirurgia ortopédica. Revista Sobecc. 22(1): 17-22. https://revista.sobecc.org.br/sobecc/article/view/137

Strieder, A. T., Graube, S. L., Dezordi, C. C. M., Stumm, E. M. F. et al. Atuação do enfermeiro no processo de limpeza em um centro de material e esterilização. Rev. SOBECC. 24(1): 50-53.: https://revista.sobecc.org.br/sobecc/article/view/425 